\section{THU0732-HPR INDIVIDUAL RESPONDER ANALYSIS OF THE EFFECTIVENESS OF MANUAL THERAPY AND EXERCISE VERSUS USUAL CARE IN PATIENTS WITH CHRONIC NONSPECIFIC NECK PAIN: PRELIMINARY RESULTS OF A RANDOMISED CONTROLLED TRIAL}

L.A. Domingues ${ }^{1,2}$, F.M. Pimentel-Santos ${ }^{1,3}$, E.B. Cruz ${ }^{4}$, J.C. Branco ${ }^{1,3}$.

${ }^{1}$ Rheumatological Disease, CEDOC - Chronic Diseases Research Center - Nova Medical School / Faculdade de Ciências Médicas - Universidade Nova de Lisboa;

${ }^{2}$ Unidade de Fisioterapia, Centro de Medicina e Reabilitação de Alcoitão;

${ }^{3}$ Rheumatology Department, CHLO / Hospital Egas Moniz, Lisboa; ${ }^{4}$ Escola

Superior de Saúde - Instituto Politécnico de Setúbal, Setúbal, Portugal

Background: Chronic nonspecific neck pain (CNP) is a common health problem worldwide. The physiotherapy approach is the second line of treatment and a large variety of modalities are frequently used. However, the mean effect of interventions is small and it is unknown if the patients achieving clinically important change. Individual responder analyses provide researchers with complementary information about the patterns of recovery and the proportion of patients achieving clinically important treatment responses.

Objectives: The aim of this study was to compare the effectiveness of a combined intervention of manual therapy and exercise (MET) versus usual care (UC), on pain intensity and global perceived recovery.

Methods: A randomised controlled trial was conducted on 62 participants with CNP lasting $\geq 3$ months, assigned to MET and UC groups. Participants in the MET group $(n=31)$ received 12 sessions of passive articular mobilisation and exercise (coordination, strength, endurance), whereas the UC group $(n=31)$ received 15 sessions of usual physiotherapy care, combining electrotherapy, massage and stretching exercises. Patients were assessed at baseline, and then at 3 and 6 weeks (final of intervention). The Minimal Clinically Important Difference (MCID) in treatment response for pain intensity was defined as a decrease of $>2$ point in the Numeric Rating Scale of Pain compared to the baseline score and for global perceived recovery a value of $\geq 5$ in Patient's Global Impression of Change Scale.

Results: A significant difference between-groups was observed at 6 weeks on pain intensity ( $\leq 0.001)$, favouring the MET group. No significant differences were found between-groups in pain intensity at the baseline $(p>0.626)$ and at 3 weeks $(p \geq 0.777)$. At patient-level response, in the MET group, $58 \%$ of the participants experienced an MCID in the first 3 weeks of treatment and this proportion was increased to $94 \%$ at 6 weeks on pain intensity, and rose from $68 \%$ to $81 \%$ on global perceived recovery. In the UC group the proportion of patients that experienced an MCID rose from $55 \%$ to $61 \%$ on pain intensity, at 3 and 6 weeks, respectively, and $68 \%$ in global perceived recovery in both moments. The patients in MET group were $10 \%(R R=1,1)$ and $50 \%(R R=1,5)$ more likely to achieve the MCID on pain intensity than the UC group, at 3 and 6 weeks, respectively. In global perceived recovery, the MET group were $20 \%(R R=1,2)$ more likely to achieve an MCID response at the 6 weeks. No differences were found in chances of recovery at 3 weeks.

Conclusions: These findings suggest that participants of MET group had a pattern of recovery over 6 weeks and achieved a higher response rate to treatment, on pain intensity and a better global perceived recovery, compared to those receiving UC

Disclosure of Interest: None declared

DOI: 10.1136/annrheumdis-2018-eular.3693

\section{THU0733-HPR EFFECT OF CUSTOM-MADE FOOT ORTHOSES VERSUS PLACEBO IN PATIENTS WITH RHEUMATOID ARTHRITIS: RANDOMISED CLINICAL TRIAL}

M. Reina-Bueno $^{1}$, P.V. Munuera-Martínez ${ }^{2}$, C. Vázquez-Bautista ${ }^{1}$, S. PérezGarcía ${ }^{3}$, C. Rosende-Bautista ${ }^{3} .{ }^{1}$ Podiatry, University of Seville, Seville; ${ }^{2}$ Podiatry, University of Seville, Sevilla; ${ }^{3}$ Ciencias de la Salud, University of a Coruña, Ferrol, Spain

Background: Rheumatoid Arthritis (RA) affects among $0.3 \%$ and $1.5 \%$ of people. ${ }^{1}$ Foot involvement occurs in more than $85 \%$ of people with RA. ${ }^{2}$ One of the most prevalent deformities is rearfoot valgus. ${ }^{3}$

Objectives: To study whether the use of custom made foot orthoses improves pain, foot function, and quality of life in RA patients.

Methods: This randomised clinical trial was carried out in the University of Seville and of A Coruña. Inclusion criteria: to be over 18 years and to have diagnosis of RA with involvement in the foot. Exclusion criteria: Acute phase, neurological problems or cognitive impairment. Participants were given an informational form. They had to use physiological footwear and assigned foot orthoses during at least 8 hours/day, for 3 months. Participants were randomly assigned to one of the two groups: Experimental Group (A) (foot orthosis with a Rovalfoam upper sheet and polypropylene) and Control Group (B) (5mm-thick Rovalfoam sheet, without adaptation). The SF-12, the Visual Analogue Pain Scale (EVA), the Manchester
Questionnaire and the Foot Function Index (FFI) are administered at the first visit and at the last review.

Results: The final sample consisted of 47 participants with RA, $53.2 \%$ were group A and $46.8 \%$ were group $B$. This

Abstract THU0733HPR - Table 1. shows the results of the different questionnaires for both groups.

\begin{tabular}{|c|c|c|c|c|c|}
\hline & & \multicolumn{2}{|c|}{ Group A } & \multicolumn{2}{|c|}{ Group B } \\
\hline & & Baseline & End & Baseline & End \\
\hline EVA & & $6.52 \pm 2.34$ & $4.16 \pm 2.82$ & $6.23 \pm 1.88$ & $5.47 \pm 2.89$ \\
\hline \multirow[t]{5}{*}{ Manchester } & Functional & $12.32 \pm 5.77$ & $10.72 \pm 7.17$ & $12.19 \pm 4.53$ & $10.71 \pm 5.58$ \\
\hline & $\begin{array}{l}\text { Personal } \\
\text { Appearance }\end{array}$ & $1.2 \pm 1.44$ & $0.68 \pm 1.21$ & $1.62 \pm 1.56$ & $1.38 \pm 1.57$ \\
\hline & Pain & $7.00 \pm 2.61$ & $5.52 \pm 3.28$ & $6.52 \pm 3.06$ & $5.47 \pm 2.63$ \\
\hline & Work & $2.56 \pm 1.78$ & $2.12 \pm 1.83$ & $2.33 \pm 1.46$ & $2.19 \pm 1.75$ \\
\hline & Total & $22.60 \pm 9.58$ & $\begin{array}{c}19.04 \\
\pm 11.93\end{array}$ & $21.86 \pm 8.54$ & $19.76 \pm 9.79$ \\
\hline \multicolumn{6}{|l|}{$\mathrm{FFI}$} \\
\hline & Pain & 69.26 & 45.97 & 61.85 & 53.36 \\
\hline & & \pm 18.67 & \pm 29.11 & \pm 19.59 & \pm 25.95 \\
\hline & Disability & 58.97 & 45.55 & 57.71 & 46.67 \\
\hline & & \pm 28.95 & \pm 34.43 & \pm 24.09 & \pm 30.97 \\
\hline & Activity & 24.75 & 13.24 & 14.14 & 10.54 \\
\hline & Limitation & \pm 23.76 & \pm 14.67 & \pm 12.43 & \pm 10.55 \\
\hline & Total & 54.70 & 39.08 & 51.12 & 42.69 \\
\hline & & \pm 20.55 & \pm 26.26 & \pm 15.98 & \pm 24.17 \\
\hline
\end{tabular}

At the initial moment between groups, there were no statistically significant differences.

Data obtained baseline and end of the follow-up period were compared within the control group, and showed significant differences in some FFI domains (days with pain, disability and total). In the experimental group there was a statistically significant decrease in EVA scale, some Manchester questionnaire domains (pain and total sections) and in some FFI domains (activity limitation and total; there was a very statistically significant in EVA scale and in some FFI domains (pain and total). There was no significant difference in the SF-12 scale between these two moments.

Conclusions: Custom made foot orthoses improved foot pain and function in people with RA who participate in this study. However, this treatment did not have a positive effect on their quality of life.

\section{REFERENCES:}

[1] Novak P, et al. Influence of foot orthoses on plantar pressures, foot pain and walking ability of rheumatoid arthritis patients-a randomised controlled study. Disabil Rehabil 2009;31(8):638-45.

[2] Bongi SM, et al. A comparison of two podiatric protocols for metatarsalgia in patients with rheumatoid arthritis and osteoarthritis. Clin Exp Rheumatol 2014:32:855-63.

[3] Barn R, et al. Kinematic, kinetic and electromyographic response to customized foot orthoses in patients with tibialis posterior tenosynovitis, pes plano valgus and rheumatoid arthritis. Rheumatol 2014;53(1):123-30.

Disclosure of Interest: None declared

DOI: 10.1136/annrheumdis-2018-eular.3421

\section{THU0734-HPR NOVEL EXPERIENCE EQUIPMENT FOR RHEUMATOID HAND-FINGERS}

M. Kusumoto ${ }^{1}$, S. Tsuji ${ }^{2}$, M. Toshiharu ${ }^{3}$, Y. Kushimoto ${ }^{1}$, N. Ise ${ }^{1}$, N. Yoshida ${ }^{1}$ S. Kawai' ${ }^{1}$ T. Kyrosawa ${ }^{3}$, E. Oguro ${ }^{2}$, Y. Okita ${ }^{3}$, K. Kuzuya ${ }^{2}$, H. Matsuoka ${ }^{2}$, S. Teshigawara ${ }^{2}$, M. Yoshimura' ${ }^{2}$, Y. Harada ${ }^{2}$, T. Noguchi ${ }^{2}$, M. Matsushita ${ }^{2}$ K. Hagio ${ }^{1}$, S. Tsuji ${ }^{2}$, S. Akita ${ }^{2}$, S. Ohshima ${ }^{2}$, J. Hashimoto ${ }^{2}$, Y. Saeki' ${ }^{2}{ }^{1}$ Department of Rehabilitation; ${ }^{2}$ Department of Rheumatology, National Hospital Organization Osaka Minami Medical Center, Kawachinagano, ${ }^{3}$ Kawamura Gishi Co., LTD. Daito, Japan

Background: RA patients are prone to ulnar deviation and swan-neck deformity even early after onset of the disease. Limitation of finger joint range of motion due to hand-finger deformation brings restriction to $A D L$ in the workplace as well as in the home. Patients and caretakers of patients are often burdened by these limitations; however, RA hand-finger deformation experience equipment have not been developed to experience these limitations. We have developed a novel RA handfinger deformation experience equipment with opened fingertips (RSE; RA hand/ finger simulation equipment).

Objectives: To assess the utilisation of RSE in healthy volunteers (HV) to experience RA hand-finger dysfunction using DASH (Disabilities of the Arm, Shoulde 
and Hand), STEF (Simple Test for Evaluating Hand Function), and Purdue Pegboard.

Methods: We developed the following equipment: Type $U$ to imitate extension limitation of metacarpophalangeal (MCP) joints seen in ulnar deviation; Type B which imitates flexion deformity of the distal interphalangeal (DIP) joints by seen in boutonniere deformity; and Type $S$ which imitates flexion limitation of proximal interphalangeal (PIP) and interphalangeal (IP) joints by reversing the upper and lower ends of the Oval-8 Finger Splint (Fukui Co. Ltd, Japan). Types U and S were fitted on HV (index to pinky). RSE was evaluated using DASH, STEF and Purdue Pegboard in hand-finger function evaluation. Twenty-four RA patients with handfinger deformation and Forty-one HV were included in this study to evaluate the equipment.

Results: Mean \pm SD ages for RA patients was $67.4 \pm 8.0$ years (95.8\% female) and $38.2 \pm 17.7$ for HV (63.4\% female), respectively. Total hand-finger deformities for RA patients were 23 hands for ulnar deviation, 66 fingers for swan-neck deformity, and 33 fingers for boutonniere deformity. Randomization for RA patients was as follows: 13 DASH, 5 (10 hands) STEF, and 6 Purdue Pegboard. $10 \mathrm{HV}$ were assigned to DASH, 10 to Purdue Pegboard, and 14 (28 hands) to STEF. HV were evaluated with RSE and without RSE. For DASH, STEF, and Purdue Pegboard, RA patients showed significant functional loss compared to HV. Significant function loss in RA patients was also observed with the RSE. However, no differences were seen between the RA group and the HV with RSE group (figure 1).

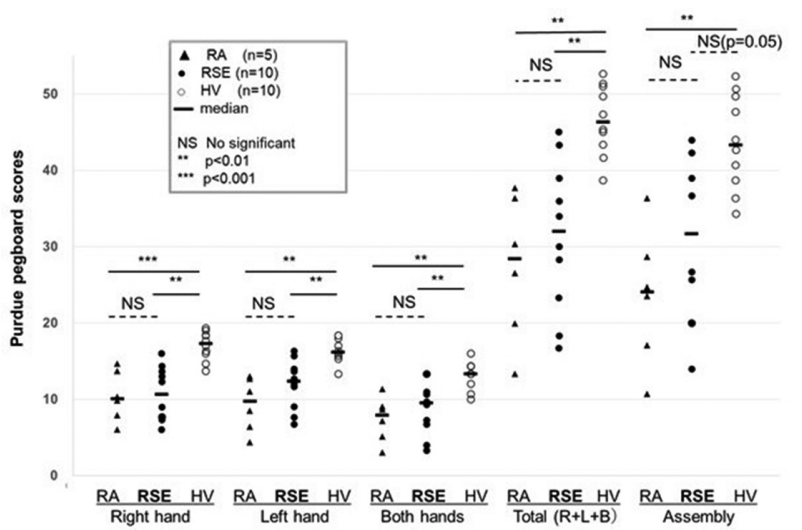

Abstract THU0734HPR - Figure 1 Purdue. pegboard scores of RA, RSE and HV groups

Conclusions: We developed the RSE, which allows for one to experience the decrease in function with RA hand-finger deformity. Our study showed that RSE use can indeed allow this experience. By using RSE, health care workers, patient caretakers and early RA patients can experience joint limitation of RA for educational purposes, personalised rehab programs, and development of self-help tools.

Disclosure of Interest: None declared

DOI: 10.1136/annrheumdis-2018-eular.3416

\section{THU0735-HPR HOW RHEUMATOLOGY SPECIALIST NURSE DETECTS SMOKING HABIT IN RHEUMATOID CHRONIC INFLAMMATORY DISEASE PACIENTS}

M. Serra Miralles ${ }^{1}$, F. Abella ${ }^{2}$, N. Montalà ${ }^{1}$, M. Mateu ${ }^{1}$, A. Palau ${ }^{1}$, M. Conde ${ }^{1}$, L. Roselló ${ }^{1}$ 'Rheumatology, ${ }^{2}$ Psychology, Hospital Santa Maria, Lleida, Spain

Background: Inflammation plays a significant role in the development of atherosclerosis and cardiovascular disease (CVD). Patients with chronic inflammatory disease (CID) have an increased risk of CVD. Smoking is one of the main risk factors for CVD and a predictor of poor response to treatment and poor prognosis.

Objectives: We aimed to describe the role on a rheumatology specialist nurse in the detection of smoking habit and dependence to nicotine in CID patients before the start of an interventionism plan to avoid it in CID patients.

Methods: All CID patients attended in a nurse rheumatologist unit, during one month, were asked for smoking habit. We determined the characteristics, onset of smoking and number of cigarettes per day. The Fagerström test was used to establish the nicotine dependence (ND) (score >5) and the Richmond test for predicting abstention following intervention to stop smoking (score $>5$ ). Exhaled $\mathrm{CO}$ level Coximetry was also recorded. We planned an interventionism consenting in health advice provided by a nurse and the derivation to a smoke unit.

Results: 22 patients were identified. $12(50.0 \%)$ were female and mean age was 46.0 (SD 10.5). 14 (63.6\%) suffered Rheumatoid Arthritis, 6 (27.3\%) Ankylosing Spondylitis and $2(9.1 \%)$ Psoriatic Arthritis. 5 (22.7\%) subjects were under biological treatment. Mean number of cigarettes smoked per day and smoking years were 16.0 (SD 8.9) cigarettes and 27.2 (SD 11.7) years respectively. Up to 5 out of $22(22.7 \%)$ subjects had high ND. Patients with ND had higher exhaled CO levels than non ND subjects (24.6 [SD 6.0] vs. 17.9 [SD 12.2] p-value 0.160). We observed no differences in age, sex, rheumatoid diagnosis, years smoking or cigarettes per day between both groups. Active treatment with biological drugs was significantly associated with ND (60.0 vs. $14.3 \%$, p-value 0.046$)$. One out of five patients with ND had good abstention prediction according to Richmond test Conclusions: Nearly one out of five smoking CID subjects had severe ND. Treatment with biological drugs was related to ND. Interestingly, most of the ND subjects had good prediction for smoking cessation. Therefore, the development of a nurse program to detect smoking and our interventionism plan to avoid smoking habit are of enormous interest.

\section{REFERENCES}

[1] Oliver S, Leary A. The value of the nurse specialists' role: Pandora initial findings. Musculoskeletal Care 2010;8:175-7.

[2] Soderlin MK, Petersson IF, Geborek P. The effect of smoking on response and drug survival in rheumatoid arthritis patients treated with their first antiTNF drug. Scand J Rheumatol 2012;41:1-9.

[3] Chang K, Yang SM, Kim SH, Han KH, Park SJ, Shin JI. Smoking and rheumatoid arthritis. Int J Mol Sci 2014;15:22279-95.

[4] Roelsgaard IK, Thomsen T, Ostergaard M, et al. The effect of an intensive smoking cessation intervention on disease activity in patients with rheumatoid arthritis: study protocol for a randomised controlled trial. Trials 2017;18:570

Disclosure of Interest: None declared

DOI: 10.1136/annrheumdis-2018-eular.4105

\section{THU0736-HPR THE EFFECTIVENESS OF SHORT TRAINING IN PSYCHOLOGICAL SUPPORT FOR NURSES IN RHEUMATIC CARE}

M. Fusama ${ }^{1}$, H. Nakahara ${ }^{2}$, K. Higashi ${ }^{1}$, M. Mori ${ }^{3}$, M. Mabuchi ${ }^{4}$, Y. Sakai ${ }^{5}$, T. Motonaga ${ }^{6}$, T. Takeuchi ${ }^{7}{ }^{1}$ Division of Nursing; ${ }^{2}$ NTT West Osaka Hospital, Osaka; ${ }^{3}$ Jikei University School of Medicine; ${ }^{4}$ Tokyo University of Agriculture and Technology, ${ }^{5}$ Atomi University; ${ }^{6}$ Teikyo University, ${ }^{7}$ Department of Internal Medicine, Keio University School of Medicine, Tokyo, Japan

Background: Patients with rheumatoid arthritis (RA) often suffer from psychological concerns. ${ }^{1}$ There is a lack of psychology specialists in many hospitals and clinics. According to EULAR, nurses should undertake a significant portion of providing psychological support to patients. ${ }^{2}$ However, in Japan nurses involved in clinical practice often do not have sufficient time and opportunity to receive adequate education on psychological support.

Objectives: The aim of this study is to evaluate nurses' understanding of basic psychological concepts and the efficacy of a short psychology workshop for nurses in RA clinical care.

Methods: A clinical psychotherapist provided a lecture on psychological support once for a period of $30 \mathrm{~min}$, followed by 1 hour of role-play groupwork. The lecture focused on basic psychological support including concepts of "listening", "acceptance", "empathy", "open question", "closed question" and "patient-centred perspective". Group work focused on applying the aforementioned concepts. Nurses were asked before and after workshop regarding their understanding and opinions on the necessity and feasibility of psychological support, as well as their motivation for implementation. Nurses' opinions were evaluated on a 1-7 rating scale (1=not at all, 7=full). Client Satisfaction Questionnaire-8 (CSQ-8J) was utilised to assess satisfaction with the workshop. Data analyses were performed with Wilcoxon signed rank test.

Results: 53 nurses (M/F: 1/52) participated in this study. The average of ages clinical experience and clinical experience in RA care were 45.5 years old, 21.0 and 7.9 years, respectively. Nurses' opinions regarding the necessity and feasibility of psychological support in general, as well as their motivation for providing psychological support statistically significantly increased post-workshop (necessity: $p=0.0052$, feasibility: $p<0.0001$, motivation: $p=0.0033$ )

Nurses' answers regarding their current application of these concepts in clinica practice were as follows: mean $\pm S D$; listening: $5.15 \pm 1.20$, empathy: $5.06 \pm 1.18$, acceptance: $4.71 \pm 1.18$, open question: $4.80 \pm 1.31$, closed question: $4.49 \pm 1.47$. The understanding of these concepts was statistically significantly higher after the workshop ( $p<0.0001$ for each concept). Moreover, the feasibility of all the above concepts was rated as statistically significantly higher post-workshop. $(p<0.001$ for each concept). The necessity of adopting a patient centred perspective was also judged as statistically significantly higher post-workshop $(p<0.001)$. Based on CSQ-8J, satisfaction with the workshop was overall high.

Conclusions: This is the first report evaluating the effectiveness of brief psychological workshops for nurses in rheumatic care in Japan. This preliminary study 